\title{
Scenario-based Prediction-of-Events: A New Theory Linking Strategic Planning of Healthcare Operations to the Prognosis of Medical Conditions
}

Soliman $\mathbf{R}^{*}$, Eweida W, Zamzam M and Abouelnaga S

Children's Cancer Hospital, Egypt

\begin{abstract}
The "Scenario-based Prediction-of-Events" theory and conceptual model predicts the occurrence of future events according to different potential scenarios based on fixed certainties that include business, humanistic, and system-related factors, as well as other unexpected uncertainties. The new theory and model could be applied in any industry. Upon application in healthcare, the new model links strategic planning of healthcare operations to the prognosis of medical conditions. Prediction of how a certain medical condition would behave and creating scenarios based on the certainties (historical data) and the uncertainties (future expectations) using scenario-based analysis is an innovative strategic approach. The model will predict all the possible scenarios for prognosis of a medical condition and their consequences on resource utilization and strategic planning. Developing standard operating procedures (SOPs) for each written scenario with expected cost and capacity requirements will help the organization better strategically predict future needs for capacity-building and lead to better strategic planning of healthcare operations as well as management of organizational resources.
\end{abstract}

Keywords: Scenario analysis; Prediction; Strategic planning; Healthcare operations; Prognosis of medical conditions; Conceptual model

\section{Introduction}

Healthcare is a complex industry that has long suffered from a lack of coordination, high variability and failure to tie costs to quality [1]. For decades, the problem of how to allocate healthcare resources in a just and equitable fashion has been the subject of intensive discussion and analysis, yet the issue has not been resolved yet [2]. Another major problem that many healthcare organizations are facing is the unpreparedness for any unexpected situation which can lead to an economic crisis affecting the capacity utilization of the healthcare organizations and subsequently affecting the lives of many patients [3]. That is why a new strategic approach in healthcare is needed to tackle these problems and to predict upfront the expected costs and capacity requirements needed to face the changing surroundings due to intrinsic or extrinsic factors.

\section{Background and Significance}

\section{Scenario analysis}

Scenarios are stories about the future, but their purpose is to make better decisions in the present [4]. Scenarios deal with the uncertainty arising from the fact that we don't know in what kind of future today's plans and decisions will unfold, where a set of scenarios can illustrate different possible pathways into the future [4]. Scenario analysis is a process of analyzing possible future events by considering alternative possible outcomes. Thus, scenario analysis, which is a main method of projections, does not try to show one exact picture of the future. Instead, it presents consciously several alternative future developments. Consequently, a scope of possible future outcomes is observable [5]. Scenario analysis is designed to allow improved decision-making by allowing consideration of outcomes and their implications. Scenario analysis is the merging of two worlds: the past and the present to predict the future [6].

\section{Active management of healthcare operations}

Due to the high costs involved in healthcare delivery and the forecasted increase in the demand, efficient allocation of healthcare resources has become more crucial. Operations management is the design, operation, and improvement of the processes and systems that create and deliver the organization's products and services. The goal of operations management is to more effectively and efficiently produce and deliver the organization's products and services [7]. A new approach is the Active Management of Healthcare Operations (AMHO) which is a framework for healthcare decision-making and management of healthcare operations through the application of dynamic resource management (DRM) using information technology [7]. The AMHO approach captures factors that might influence how proposed plans and current activities unfold and provides visibility into the range of possible outcomes. Examples of such capabilities include resource projection, predictive management, emergency planning and real time response (situational awareness), as well as financial forecasting [7].

\section{Scenario thinking and strategic planning}

Scenario planning or scenario thinking is a strategic planning tool used to make flexible long-term plans. It is a method for learning about the future by understanding the nature and impact of the most uncertain and important driving forces affecting our world [8]. Scenario planning has been found to be in the heart of intersection and overlap between organizational strategy, environmental analysis and forecasting [8]. Strategic planning requires an understanding of future developments in the environment in which their decisions will be made. However, there is increasing recognition that there is no single predetermined "future." Therefore, the use of alternative future scenarios can be helpful. Scenario construction is a technique for combining possible environmental developments in a systematic way to assess the

*Corresponding author: Ranin Soliman, Assistant Chief Operating Officer Children's Hospital Egypt (57357 Hospital), Egypt, Tel: +201003263399; E-mail: raninmagdi@hotmail.com

Received January 23, 2017; Accepted February 21, 2017; Published February 28, 2017

Citation: Soliman R, Eweida W, Zamzam M, Abouelnaga S (2017) Scenario-based Prediction-of-Events: A New Theory Linking Strategic Planning of Healthcare Operations to the Prognosis of Medical Conditions. Health Care Current Reviews 5: 190. doi: 10.4172/2375-4273.1000190

Copyright: (c) 2017 Soliman R, et al. This is an open-access article distributed under the terms of the Creative Commons Attribution License, which permits unrestricted use, distribution, and reproduction in any medium, provided the original author and source are credited. 
potential consequences of alternative decisions [9]. The value of scenario planning lies in how scenarios are embedded providing vital links between organizational processes such as strategy making, innovation, risk management, and leadership development [10].

\section{Scenario planning in healthcare}

Facing future developments in health care, scenario planning offers a complementary approach to traditional strategic planning [1]. Traditional strategic planning consists of predicting the future at a single point on a chosen time horizon and mapping the preferred plans to address such a future [10]. On the other hand, scenario planning creates stories about multiple likely potential futures on a given time horizon and maps the preferred plans to address the multiple described potential futures [10]. It identifies specific forces affecting healthcare delivery systems, tests assumptions for the future, assesses alternative futures and can form a foundation for future planning [11]. Scenarios are particularly useful in a rapidly changing world with shifting external conditions such as healthcare systems [12].

\section{Aim of Work}

The purpose of this paper is to introduce a new theory applying scenario-based prediction-of-events in strategic planning of healthcare operations in order to better manage healthcare costs and resources and increase the efficiency of utilization. The theory presents a conceptual model that predicts the incidence of events in the prognosis of medical conditions according to different potential scenarios, based on a number of influencing factors.

\section{Materials and Methods}

\section{Study design and data sources}

A systematic qualitative literature review was done for sources whose focus was on the process of scenario planning and/or scenario analysis. This was followed by thematic analysis of the findings to aggregate the concepts into themes related to the building blocks of scenarios and the domains or elements that affect the process of scenario planning.

\section{Selection of factors affecting scenario planning and analysis}

The model is based on the components of scenario planning which divides our knowledge into two broad domains: (1) things we believe we know something about (certainties) and (2) elements we consider uncertain (uncertainties), at a certain point of time [13]. The first component - trends and certainties- casts the past forward, recognizing that our world possesses considerable momentum and continuity, such as making assumptions about prognosis of a medical condition based on previous historical data and trends [13]. The second component true uncertainties - involves the indeterminables such as future success rates, and outcomes of medical condition prognosis [13]. The art of scenario planning lies in blending the known and the unknown into a limited number of internally consistent views of the future that span a very wide range of possibilities. The third factor added is the time, because scenarios must pertain to a meaningful duration of time, for the passage of time will make the scenarios obsolete [3].

\section{Building blocks for scenarios}

The building blocks for scenarios include drivers of change, basic trends, key uncertainties, rules of interaction, leading to multiple scenarios [13].

\section{Process for building scenarios}

The model was built following the five-phase scenario analysis process [14] as follows:

1. Defining the problem and what needs to be achieved at a specific time horizon.

2. Identifying the key factors, trends, and uncertainties that may affect the plan.

3. Separating certainties from uncertainties.

4. Developing scenarios by making multiple interactions between certainties and uncertainties.

5. Using the scenarios in strategic planning for the future.

\section{Results}

\section{Outcome: A new theory and conceptual model}

By combining the concepts of scenario-analysis and scenario-planning with the concepts of healthcare operations management for a specific medical condition or disease, a new theory evolved. The new theory and conceptual model statement that was created by the authors of this article is as follows: "Planning scenarios for all the predictable pathways, deviations and prognosis of medical conditions helps to accurately plan in advance the needed capacity and resource utilization, and the forecasted costs for each potential scenario. The prediction of events for medical conditions is based on the estimate certainties from historical data and other uncertainties that may happen in the future. This allows for better strategic planning and organizational preparedness to better forecast future capacity- building and resource utilization in a systematic healthcare operations management framework". This theory does not consider the incidence of an event as the deviation from the usual scenario, but rather deals with the occurrence of the different events in the prognosis of medical conditions as predicted scenarios with varying likelihoods of occurrence. Based on this theory, a new conceptual model was developed to apply the methods of scenario analysis in the prediction of future events that affect prognosis of medical conditions and thus affect the future resource utilization capacity and projected cost estimates. The conceptual model was developed to predict the occurrence of future events according to different potential scenarios based on fixed certainties (business, humanistic, system-related factors) and other unexpected uncertainties (political, economic, environmental) through a systemic process of scenario-analysis and planning.

\section{Probability-of-Events Concept: A Novel Concept about Life and Death}

Since a theory and conceptual model were proposed to predict all the possible future scenarios regarding the probability of events in healthcare (prognosis of medical conditions), then "death" was viewed as one of the predicted scenarios/outcomes of the normal progression of any medical condition. Death should be placed as one of the possible probabilities to happen as a result of the progression of any medical condition. Whereas, most healthcare professionals and experts view death due to natural progression of a medical condition as an unpredicted event. The clinicians, physicians and the healthcare team need to put in mind that "death" is a normal probability for the progression of medical condition or disease and that it should be dealt with as a predicted potential scenario, rather than a deviation from the normal scenario. After all, "death" is a certainty that cannot be denied. The factor of "time" is the only variable factor when predicting such a scenario. 
Citation: Soliman R, Eweida W, Zamzam M, Abouelnaga S (2017) Scenario-based Prediction-of-Events: A New Theory Linking Strategic Planning of Healthcare Operations to the Prognosis of Medical Conditions. Health Care Current Reviews 5: 190. doi: 10.4172/2375-4273.1000190

Page 3 of 5

\section{Scenario-based Prediction-of-Events for Medical Con- ditions: Conceptual Model}

By applying business concepts of scenario analysis and scenario planning to the prognosis of medical conditions, the scenario-based prediction-of-events model was developed for medical conditions. For any medical condition, there is a number of predictable scenarios for prognosis of the medical conditions and how they will proceed. There are several possible situations, usually worst-case scenario, most-likely case scenario, and the best-case scenario (zero-base or zero-sum scenario).

\section{Steps for Building the Scenario-Based Prediction-of- Events Model}

- Write all possible scenarios for prognosis of the medical condition with detailed classes and levels for each scenario based on historic certainties (Givens based on basic trends).

- Write scenarios for prognosis of the medical condition with detailed classes and levels for each scenario for all possible future unpredictable deviations that may arise to each scenario (key uncertainties).

- Predict multiple interactions between influencing factors leading to multiple scenarios in prognosis of medical conditions [13].

- Assign frequency rates for the occurrence of each scenario (though they are just estimate values depending on historic data and may change based on unpredictable deviations, but can give predictable ranges for likelihood of occurrence).

\section{“Zero-base" scenario}

A Zero-base Scenario is the baseline pathway for any process of product or service development without any external influencing factors, bearing zero pressure without any deviation from the ideal bestcase scenario.

\section{Deviation from "zero-base" scenario}

Deviations from zero-based scenarios will be based either on Cer- tainties (Givens) or Unpredictable Uncertainties at any point of time (Tn). These deviations are the building blocks of the model (Figure 1).

\section{Building Blocks of the Conceptual Model}

\section{A. Predictable influencing factors (certainties)}

These are predictable events anticipated from historic data that can occur and shift the normal path of the prognosis of the medical condition. They include

\section{Business-related factors (B)}

These are the influencing factors that are related to the core product or service of any business model or industry. These Business-related factors are generic factors that include the product-related factors and the servicerelated factors, depending on the type of industry and the scope of business.

These product- or service-related business factors can be applied to any business model or industry. In the healthcare industry and the delivery of health care services, the main influencing factors are the medical condition-related factors -also referred to as 'disease-related factors'- that greatly impact the type, frequency and variety of the delivered health care services.

These disease-related factors (D). That can affect the prognosis of the disease or medical condition, either as initial contributing factors of the disease or the patients, or disease-related factors during the course of management and treatment of the disease. The disease-related factors take into account the severity of the disease or medical condition (stage, grade, or risk), degree of response to treatment, disease-related or treatment-related complications, and evidence of disease recurrence).

\section{Humanistic factors $(\mathrm{H})$}

These are the influencing factors that may arise due to personal variations in the delivery of services. They may include non-compliance to treatment, medical errors, medication errors, procedural errors, wrong diagnosis or stratification, delays due to individual-related factors.

\section{System-related factors (S)}

These are the factors related to systems issues that can arise and

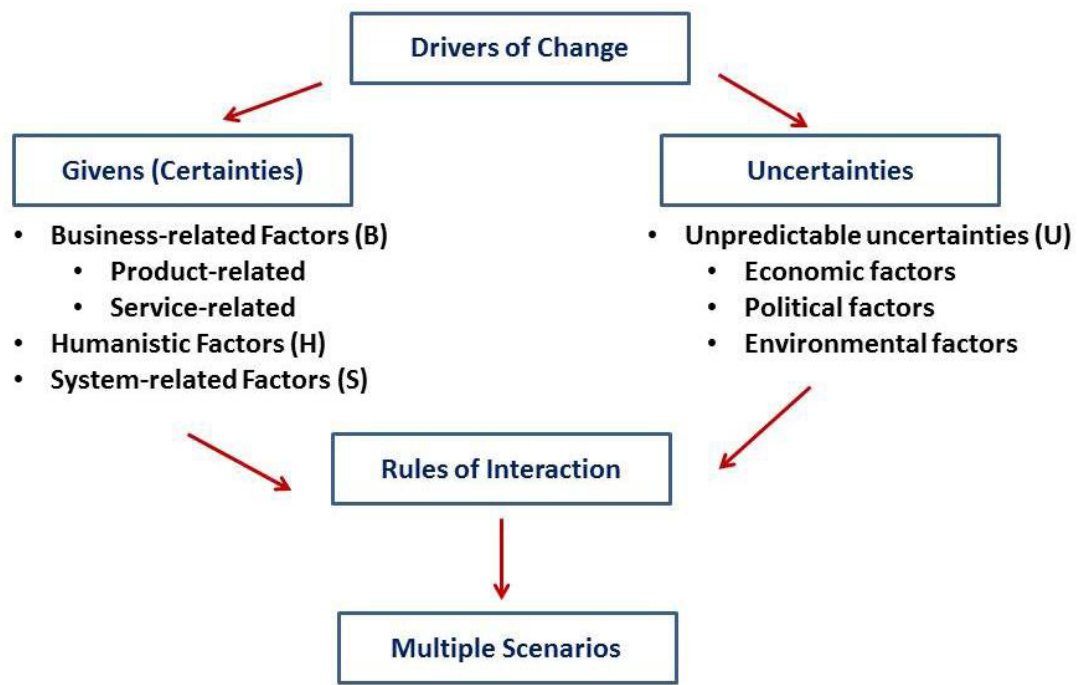

Figure 1: Building blocks of the scenario-based prediction-of-events model. 
Citation: Soliman R, Eweida W, Zamzam M, Abouelnaga S (2017) Scenario-based Prediction-of-Events: A New Theory Linking Strategic Planning of Healthcare Operations to the Prognosis of Medical Conditions. Health Care Current Reviews 5: 190. doi: 10.4172/2375-4273.1000190

affect the prognosis of the disease. These may include: Delays due to waiting list or workflow, diagnostic tool error, scheduling delays, or delays related to workflow issues during transfer or discharge.

\section{B. Unpredictable influencing factors (uncertainties)}

These are the external factors that can lead to deviation from zero-base scenario of disease prognosis that could not be expected before. These uncertainties $(\mathrm{U})$ include economic, political or environmental factors.

\section{Timing of occurrence of event or deviation}

The timing of the event is an independent variable that affects the prognosis of the disease leading to different scenarios at varying timings of the event. This is known as "Time-limited Event", where variation in the time of occurrence of the event leads to a different type of deviation or shifting in disease prognosis, leading to different scenarios for the same influencing factors at different points of time (Tn).

Predictability of occurrence rate can be determined in case of scenarios that deviate from zero-base according to the predictable influencing factors (Certainties), depending on historic data.

\section{Multiple Interactions and Multiple Scenarios (BHS-U at Tn Model)}

Multiple interactions between the different predictable certainties -business, humanistic, and system- (BHS) and the uncertain influencing factors $(\mathrm{U})$ at different points of time (Tn), lead to multiple scenarios with several scenario levels, classes or sub-classes (The BHS-U at Tn Model) as shown in (Figure 1).

\section{Algorithm for Scenario-Prediction-of-Events Model for Medical Conditions}

In the healthcare business, the delivery of health care services is significantly affected by the nature of the disease or medical condition. Therefore, the business-related factors would be substituted with a core influencing factor "The Disease", leading to the development of the "DHS-U at Tn" Model as shown in (Figure 2). In this case, the zero-base scenario is the baseline pathway for disease prognosis without the effect of any influencing factors; absence of disease-related factors, humanistic factors, or system-related factors. The model was built for a cancer diagnosis with detailed scenarios (Figure 2).

\section{Linking "Scenario-Based Prediction-of-Events" to Healthcare Operations Management}

Building such scenarios applies to all business models in any sector or industry in the process of product or service development. In the healthcare industry, building scenarios for the prognosis of any disease or medical condition and linking them to healthcare operations will help achieve the following:

\section{Costing}

Prediction of accurate costs for the possible scenarios of each medical condition.

\section{Capacity}

Prediction of all the needed capacity building resources to manage each specific scenario, class and level (eg, length of hospital stay, medications needed, personnel and healthcare professionals needed, all other hospital resources)

\section{Operations}

Prediction of day-to-day operations for each scenario and scenario sub-categories.

\section{Budgets}

Prediction of hospital budgets in the future based on predictable occurrence of each scenario (from historic data).

\section{Management}

Detailed standard operating procedure (SOPs) for all the activities and processes included in each scenario and scenario sub- categories.

\section{Planning}

Strategic planning for ultimate utilization of resources for each medical condition.

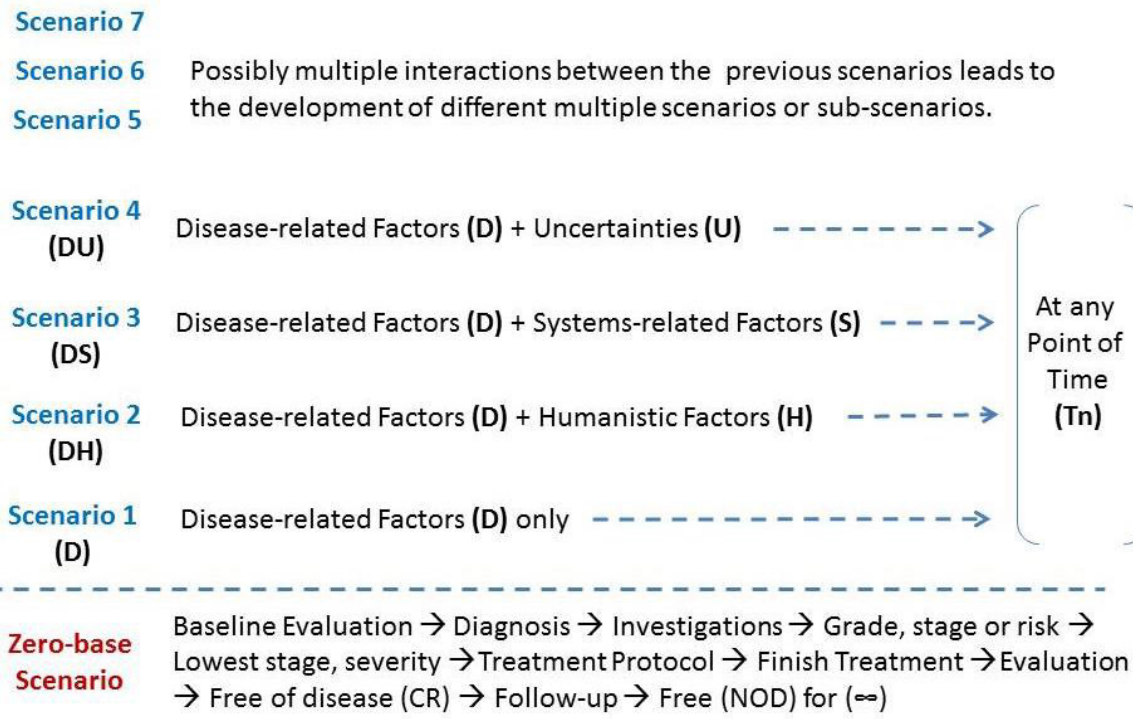

Figure 2: The algorithm of the conceptual model "DHS-U at Tn". 
Citation: Soliman R, Eweida W, Zamzam M, Abouelnaga S (2017) Scenario-based Prediction-of-Events: A New Theory Linking Strategic Planning of Healthcare Operations to the Prognosis of Medical Conditions. Health Care Current Reviews 5: 190. doi: 10.4172/2375-4273.1000190

Page 5 of 5

\section{Conclusion}

Prediction of how a certain medical condition will behave and creating systematic future scenarios is a creative and innovative way to predict all the possible consequences of the prognosis of medical conditions on the consumption of the resources in a healthcare organization. Developing SOPs for each written scenario with expected cost and capacity needs will help the organizations better strategically predict future needs for capacity-building and lead to better management of healthcare operational processes and resources. The scenario-based prediction-of-events theory and conceptual model introduces a new concept for strategic planning where a crisis or the probability of an unwanted event does not exist because all events or any crisis can be predicted in the future taking into consideration all the possible interactions between the certain and uncertain influencing factors. Therefore, it will be possible to plan preventive measures ahead to counteract or mitigate the impact of any possible risk as one of the foresighted scenarios.

\section{Discalimer}

The newly developed theory and conceptual model presented in this manuscript is a key essential step towards strategic future planning in a systematic approach. The concepts and ideas of the theory and conceptual model presented in this manuscript were not inspired, generated, or copied from any other existing systems, other than those referenced within the manuscript. Any link or relation between our conceptual model and any other systems is by chance and was not intended.

\section{References}

1. Dunn L (2014) Healthcare is Complex, And We Aren't Helping by Making It More Complicated.

2. Kluge $E$ (2007) Resource Allocation in healthcare: Implications of models of medicine as a profession. Medscape General Medicine 9: 57.

3. Dutta K, Babbel D (2010) Scenario Analysis in the Measurement of Operational Risk Capital: A Change of Measure Approach. SSRN Electronic Journal.

4. Meinert S (2014) Field manual-Scenario building.

5. Aaker D (2013) Strategic market management.

6. http://www.economist.com/node/12000755

7. Moen D, Meredith L (2010) Active Management of Healthcare Operations: A Dynamic System View of Healthcare Delivery. Journal of Medical Devices 4: 027524.

8. https://www.jisc.ac.uk/full-guide/scenario-planning

9. Zentner RGelb B (1991) Scenarios: A planning tool for health care organizations. Hosp Health Serv Adm 36: 211-22.

10. Wilkinson A, Kupers (2013) Living in the Futures. Harvard Business Review.

11. Enzmann D, Beauchamp N, Norbash A (2011) Planning Scenario. Journal of the American College of Radiology 8: 175-179.

12. Stavem K (2000) Scenario analysis-a method for long-term planning. Tidsskr Nor Laegeforen 120: 100-101.

13. http://www.slideshare.net/eteigland/crash-course-in-scenario-planning

14. Schwartz $P$ (1996) The art of the long view: Paths to strategic insight for yourself and your company. New York: Random House. 